sage $\mathrm{zu}$ bewegen, dass auch nach seiner Auffassung nicht per se alles erlaubt sei, sondern dass der Staat quasi aus Selbstachtung auch den Feind menschenwürdig behandeln müsse. Hierzu zwingt ihn aber nach Jakobs' Auffassung - dann nicht mehr das Straf- oder Strafprozessrecht.

Die Diskussion war durch diesen Beitrag sehr stark geprägt, so dass andere wichtige Aspekte, die auch in den Arbeitsgruppen bedeutsam waren, nur noch am Rande Aufmerksamkeit fanden. Sowohl Griesbaum als auch Haffke wiesen aber an verschiedenen Stellen darauf hin, dass einer Entwicklung, die lediglich
Straf- und Strafprozessrecht "rein « hält von Gefahrenabwehr und diese dem Polizeirecht überlässt, mit Sorge $\mathrm{zu}$ betrachten ist. Wer tatsächlich fordert, für die »gefährlichen Normalen « oder andere problematische Gruppen spezielle Gesetze zu schaffen - dies klang in den Diskussionen teilweise an -, wird seine Mandanten demnächst eben nicht mehr in U-Haft oder im Strafvollzug aufsuchen, sondern in Polizeihaft. Es ist dann aber zu befürchten, dass die Rechtsgarantien schwächer sind als die des Straf- und Strafprozessrechts. Insofern muss es darum gehen, staatliche Eingriffe insgesamt auf das notwendige Minimum zu beschränken, die systemische Gesamtschau, die dazu notwendig und die mit Haffke zu fordern ist, ist interdisziplinär zu leisten.

Dr. Christine Morgenstern ist wissenschaftliche Mitarbeiterin am Lehrstuhl für Kriminologie der Ernst-Moritz-Arndt-Universität Greifswald

\section{Fußnoten:}

1 Der Vortrag ist als pdf-Datei abrufbar unter www.strafverteidigervereinigungen.de.

2 Die Diskussionen fanden nicht nur in der Fachliteratur, sondern auch in den Feuilletons der FAZ, der ZEIT, der Süddeutschen Zeitung oder der Frankfurter Rundschau statt. Einen guten Überblick gewinnt man auch, wenn man die
Debatte in der Deutschen Zeitschrift für Philosophie (Jg. 52, 2004), an der u. a. Wolf Singer, Gerhard Roth und Jürgen Habermas beteiligt waren, verfolgt.

3 Abrufbar unter http://www.hrrstrafrecht.de/hrr/archiv/04-03.

4 Jakobs a.a.o.; außerdem in: Eser/ Hassemer/Burkhardt (Hrsg.): Die Deutsche Strafrechtswissenschaft vor der Jahrtausendwende. Rückbesinnung und Ausblick. München 2000, S. 47 ff. Die kritischen Auseinandersetzungen sind inzwischen zahlreich; auch sie haben teilweise den Weg in die überregionalen Tageszeitungen gefunden, vgl. z. B. Prantl in der Suddeutschen Zeitung vom 5./6.3.2005, S. 17; Lüderssen in der FR vom 28.1.2002; auch Düx ZRP 2003, S. $189 \mathrm{ff}$.

\title{
Neuregelungen des Menschenhandels - bewusste Regelung oder Anpassung an europäische Vorgaben in normativer Unfreiheit?
}

Monika Frommel

In der letzten Aprilwoche wurde vor dem Bundesverfassungsgericht über den Europäischen Haftbefehl verhandelt. Der Berichterstatter Udo Di Fabio überraschte durch eine geradezu frappierende Offenheit. Der deutsche Gesetzgeber sei keinesfalls gezwungen - sozusagen in normativer Unfreiheit - alles zu ratifizieren und umzusetzen, was in einer europäischen Richtlinie als Vorgabe beschlossen worden sei. Der deutsche Gesetzgeber sei im Bereich des Strafund Strafverfahrensrechts nicht so stark an europäische Vorgaben gebunden wie im europäischen Zivilrecht, sondern stimme der Sache nach lediglich einem völkerrechtlichen Vertrag zu, da der Zweck der EU eine Wirtschaftseinheit sei und gerade nicht ein mit Zwangsgewalt ausgestatteter Bundesstaat. Wir werden sehen, wie diese Debatte weiter gehen wird. Jedenfalls wirft sie ein grelles Licht auf die ungute Übung seit einiger Zeit, europäische Rahmenbeschlüsse »durchzuwinken«. Bei den auf der nächsten Seite abgedruckten Neuregelungen des Menschenhandels ist der Widerspruch zum deutschen ProstG 2002, das schließlich eine Legalisierung der Prostitution mit sich gebracht hat, offenkundig; denn wie kann die Vermittlung erwachsener Menschen in die Prostitution strafbar sein, wenn sie als Vertragspartner Rechte und Pflichten übernehmen können (bei den unter 21-Jährigen verlangt § 232 nicht mehr wie die Qualifizierung in $\S 180$ b Abs. 2 Nr. 2 StGB alt die Kenntnis einer Zwangslage). Friedrich Christian Schroeder hat in der NJW $(20 / 2005,1393)$ ausführlich die Kompliziertheit des europäischen Rahmenbeschlusses und deren Umsetzung beschrieben. Offenbar fällt es schwer, beim Thema Frauen- und Kinderhandel einen klaren Kopf zu behalten und die Be- troffenen nicht nur als Opfer (was sie sein können), sondern auch als Akteure zu begreifen, welche auf riskante Weise versuchen, aus einer hoffnungslosen in eine ausbaufähige wirtschaftliche Lage zu gelangen. Auch das Schweizer Recht (vgl. insoweit SZK 1/2005) folgt dem schlechten europäischen Vorbild (allerdings trieft die frühere Regelung vor Moralisierungen) und auch das österreichische Recht fällt durch Überregulierung im Strafrecht auf, allerdings regelt es den gesamten Komplex des Menschenhandels im Sexualstrafrecht, was den Verdacht aufkommen lässt, man möchte nun doch wieder zu Regulierungen von »Unzucht « zurückkehren. Es war zu befürchten, dass die Kontrolle grauer und schwarzer Märkte zu unklarem Strafrecht führen würde, aber etwas mehr Konsequenz hätte ich mir von einer europäischen und natio- nalen Gleichstellungspolitik erwartet. So kann ich nur einen Schulterschluss mit Konservativen feststellen, welche nicht an besseren Verhältnissen für Prostituierte interessiert sind, sondern der Straflosigkeit der bloßen Förderung der Prostitution nachtrauern und sich nun diese polizeilichen verlorenen Befugnisse über das Ausländerrecht, flankiert von einem neuen Strafrecht, wieder verschaffen. Damit sind die "Lichtblicke in der Schattenwelt « (so die SZ vom 23.05.2005, Die Seite Drei) wieder verdunkelt; und zwar nicht nur deswegen, weil nach der Wahl in NRW Netzwerke wie in Dortmund ins Hintertreffen geraten und eine tiefschwarze Politik wie die der Bayerischen Polizeibehörden näher rückt, welche bekanntlich besonders gerne die rotgrüne Rathausmehrheit mit ihrer CSU-Politik im Bereich der Landeszuständigkeiten ärgern. 


\section{St ̈̈̈ vom 19.02.2005}

\section{$\S 232$}

\section{Menschenhandel zum Zwecke der sexuellen Ausbeutung}

(1) Wer eine andere Person unter Ausnutzung einer Zwangslage oder der Hilflosigkeit, die mit ihrem Aufenthalt in einem fremden Land verbunden ist, zur Aufnahme oder Fortsetzung der Prostitution oder dazu bringt, sexuelle Handlungen, durch die sie ausgebeutet wird, an oder vor dem Täter oder einem Dritten vorzunehmen oder von dem Täter oder einem Dritten an sich vornehmen zu lassen, wird mit Freiheitsstrafe von sechs Monaten bis zu zehn Jahren bestraft. Ebenso wird bestraft, wer eine Person unter einundzwanzig Jahren zur Aufnahme oder Fortsetzung der Prostitution oder zu sonst in Satz 1 bezeichneten sexuellen Handlungen bringt.

(2) Der Versuch ist strafbar.

(3) Auf Freiheitsstrafe von einem Jahr bis zu zehn Jahren ist zu erkennen, wenn

1. das Opfer der Tat ein Kind ( $\$ 176$ Abs. 1) ist,

2. der Täter das Opfer bei der Tat körperlich schwer misshandelt oder durch die Tat in die Gefahr des Todes bringt oder

3. der Täter die Tat gewerbsmäßig oder als Mitglied einer Bande, die sich zur fortgesetzten Begehung solcher Taten verbunden hat, begeht.

(4) Nach Absatz 3 wird auch bestrafft, wer

1. eine andere Person mit Gewalt, durch Drohung mit einem empfindlichen Übel oder durch List zur Aufnahme oder Fortsetzung der Prostitution oder zu sonst in Absatz 1 Satz 1 bezeichneten sexuellen Handlungen bringt oder

2. sich einer anderen Person mit Gewalt, durch Drohung mit einem empfindlichen Übel oder durch List bemächtigt, um sie zur Aufnahme oder Fortsetzung der Prostitution oder zu den sonst in Absatz 1 Satz1 bezeichneten sexuellen Handlungen zu bringen.

(5) In minder schweren Fällen des Absatzes 1 ist auf Freiheitsstrafe von drei Monaten bis zu fünf Jahren, in minder schweren Fällen der Absätze 3 und 4 ist auf Freiheitsstrafe von sechs Monaten bis zu fünf Jahren zu erkennen.

\section{$\S 233$}

\section{Menschenhandel zum Zweck der Ausbeutung der Arbeitskraft}

(1) Wer eine andere Person unter Ausnutzung einer Zwangslage oder der Hilflosigkeit, die mit ihrem Aufenthalt in einem fremden Land verbunden ist, in Sklaverei, Leibeigenschaft oder Schuldknechtschaft oder zur Aufnahme oder Fortsetzung der Beschäftigung bei ihm oder einem Dritten zu Arbeitsbedingungen, die in einem auffälligen Missverhältnis zu den Arbeitsbedingungen anderer Arbeitnehmerinnen oder Arbeitnehmer stehen, welche die gleiche oder eine vergleichbare Tätigkeit ausüben, bringt, wird mit Freiheitsstrafe von durch Drohung mit einem empfindlichen Übel oder durch sechs Monaten bis zu zehn Jahren bestraft. Ebenso wird bestraft, wer eine Person unter einundzwanzig Jahren in Sklaverei, Leibeigenschaft oder Schuldknechtschaft oder zur Aufnahme oder Fortsetzung einer in Satz 1 bezeichneten Beschäftigung bringt.

(2) Der Versuch ist strafbar.

(3) § 232 Abs. 3 bis gilt entsprechend.

\section{$\S 233 a$}

\section{Förderung des Menschenhandels}

(1) Wer einem Menschenhandel nach § 232 oder $\S 233$ Vorschub leistet, indem er eine andere Person anwirbt, befördert, weitergibt, beherbergt oder aufnimmt, wird mit Freiheitsstrafe von drei Monaten bis zu fünf Jahren bestraft.

(2) Auf Freiheitsstrafe von sechs Monaten bis zu zehn Jahren ist zu erkennen, wenn

1. das Opfer der Tat ein Kind ( $§ 176$ Abs. 1) ist,

2. der Täter das Opfer bei der Tat körperlich schwer misshandelt oder durch die Tat in

Die Gefahr des Todes bringt oder

3. der Täter die Tat mit Gewalt oder durch Drohung mit einem empfindlichen Übel oder gewerbsmäßig oder als Mitglied einer Bande, die sich zur fortgesetzten Begehung solcher Taten verbunden hat, begeht.

(3) Der Versuch ist strafbar.

\section{St ̈̈G vom 14.07.1993}

\section{$\S 180 \mathrm{~b}$}

\section{Menschenhandel}

(1) Wer auf eine andere Person seines Vermögensvorteils wegen einwirkt, um sie in Kenntnis einer Zwangslage zur Aufnahme oder Fortsetzung der Prostitution zu bestimmen, wird mit Freiheitsstrafe bis zu fünf Jahren oder Geldstrafe bestraft. Ebenso wird bestraft, wer auf eine andere Person seines Vermögensvorteils wegen einwirkt, um sie in Kenntnis der Hilflosigkeit, die mit ihrem Aufenthalt in einem fremden Land verbunden ist, zu sexuellen Handlungen zu bringen, die sie an oder vor einer dritten Person vornehmen oder von einer dritten Person an sich vornehmen lassen soll. (2) Mit Freiheitsstrafe von sechs Monaten bis zu zehn Jahren wird bestraft, wer

1. auf eine andere Person in Kenntnis der Hilflosigkeit, die mit ihrem Aufenthalt in einem fremden Land verbunden ist,

oder

2. auf eine Person unter einundzwanzig Jahren einwirkt, um sie zur Aufnahme oder Fortsetzung der Prostitution zu bestimmen, oder sie dazu bringt, diese aufzunehmen oder fortzusetzen.

(3) In den Fällen des Absatzes 2 ist der Versuch strafbar.

\section{$\S 181$}

\section{Schwerer Menschenhandel}

(1) Wer eine andere Person

1. mit Gewalt, durch Drohung mit einem empfindlichen Übel oder durch List zur Aufnahme oder Fortsetzung der Prostitution bestimmt,

2. durch List anwirbt oder gegen ihren willen mit Gewalt, durch Drohung mit einem empfindlichen Übel oder durch List entführt, um sie in Kenntnis der Hilflosigkeit, die mit ihrem Aufenthalt in einem fremden Land verbunden ist, zu sexuellen Handlungen zu bringen, die sie an oder vor einer dritten Person vornehmen oder von einer dritten Person an sich vornehmenlassen soll, oder 3. gewerbsmäßig anwirbt, um sie in Kenntnis der Hilflosigkeit, die mit ihrem Aufenthalt in einem fremden Land verbunden ist, zur Aufnahme oder Fortsetzung der Prostitution zu bestimmen wird mit Freiheitsstrafe von einem Jahr bis zu zehn Jahren bestraft.

(2) In minder schweren Fällen ist die Strafe Freiheitsstrafe von sechs Monaten bis zu fünf Jahren.

Phänomene der Zwangsheirat sind als bes. schwerer Fall der Nötigung erfasst und der in $\mathbf{\$} \mathbf{2 3 6}$ geregelte Kinderhandel ist unverändert seit dem 1.04.2004 strafbar. 